\title{
Wie viele Auffrischungsimpfungen sind notwendig gegen die Zeckenenzephalitis FSME (Frühsommermeningoenzephalitis)?
}

\author{
A. Kind
}

Es wird darüber berichtet, dass 46 von 47 vollständig (nach 0, 1 und 12 Monaten) geimpfte Personen drei und mehr Jahre später einen signifikant positiven Titer IgG anti-FSME aufweisen. Nutzen und Risiken der FSME-Impfung werden anhand epidemiologischer Zahlen diskutiert. Die FSME-Impfung ist nach den vorliegenden Zahlen hochwirksam und reduziert das Erkrankungsrisiko etwa um den Faktor 100. Bei Vorliegen eines hohen Titers (höher als 5,0 MOC) ist nicht belegt, dass der Nutzen einer zusätzlichen Impfung grösser ist als das Risiko durch Impfkomplikationen. Die FSME-Schutzimpfung verursacht deutlich häufiger (vor allem neurologische) Komplikationen als andere Impfungen.

Korrespondenz:

Dr. med. Albert Kind

Steigstrasse 88

CH-8200 Schaffhausen

Tel. 0526258600

Fax 0526258605

E-Mail: kind@kanton.sh

\section{Ausgangslage}

Wegen meines Freizeitverhaltens gehöre ich selber zu den Risikopersonen für eine FSME-Infektion, und ich habe mich schon 1986 erstmals gegen FSME impfen lassen. Letztes Jahr wäre es nach den geltenden Empfehlungen des Impfstoffherstellers höchste Zeit geworden für eine erneute Auffrischungsimpfung. Mich hatte interessiert, ob ich von der letzten inzwischen 6 Jahre zurückliegenden Impfung noch messbare Antikörper habe, und siehe da, der IgG-Titer anti-FSME betrug das 5,2fache des Grenzwerts (5,2 MOC), und das Labor notierte den Kommentar «immun». Ich freute mich natürlich, dass ich immun bin und eine Impfung somit unnötig war. Aufgrund dieser Beobachtung wollte ich wissen, wie häufig zum Zeitpunkt einer fälligen Auffrischungsimpfung noch ein schützender Titer IgG anti-FSME vorliegt.

\section{Vorgehen}

Zusammen mit einigen Kollegen, Nachbarpraxen und Mitgliedern eines Qualitätszirkels sammelten wir Titerresultate zum Zeitpunkt einer fälligen Auffrischungsimpfung gegen FSME. Die Teilnehmer der Studie schlugen den Personen, die sich für eine Auffrischungsimpfung nach einer beliebigen Anzahl Jahren meldeten (oder die zu diesem Zweck aufgeboten wurden), vor, erst zu überprüfen, ob sie nicht immer noch immun seien. Die Teilnehmer waren frei in der Wahl des Labors. Die Titerbestimmung erfolgte damit zwar nicht standardisiert, aber man kann ersehen, ob die von verschiedenen Labors gemessenen Titer eine vergleichbare Häufigkeitsverteilung aufweisen. Es war den teilnehmenden Ärzten überlassen, ob mit dem vorliegenden Resultat die FSMEAuffrischungsimpfung dann doch durchgeführt wurde oder nicht.

\section{Kosten}

Sowohl die FSME-Impfung als auch die Titerkontrolle (nur IgG nötig, IgM nicht interessant) kosten je etwa Fr. 40.-. Falls die Impfung dann doch durchgeführt wird, sind die Kosten doppelt so hoch.

\section{Resultate}

Als erstes Resultat ergab sich eine gute Compliance für die Vorgehensweise, vor einer Auffrischungsimpfung zuerst die Immunität antiFSME zu überprüfen. Sowohl die beteiligten Ärzte wie die untersuchten Risikopersonen machten ohne weiteres mit. Es wurden total 47 Personen untersucht: 3 bis 11 Jahre nach der letzten FSME-Impfung hatten 46 von 47 (98\%) einen signifikanten Titer (>1,0 MOC). 1 von 47 hatte einen Titerwert von 0,9 MOC, also unter der Signifikanzgrenze: ein sonst gesunder sportlicher 79jähriger 4 Jahre nach der letzten FSMEImpfung (Tab. 1). Es fällt auf, dass über 50 Jahre alte Probanden deutlich tiefere Titer nach Impfung aufweisen als jüngere Personen.

\section{Arbeitshypothese}

Wenn Jahre nach einer FSME-Impfung ein nachweisbarer Titer vorhanden ist, darf doch vermutet werden, dass die betreffende Person gegenüber dem Virus in einem entscheidenden Vorteil ist. Analog der Hepatitis B nicht unbedingt vor der (vielleicht unbemerkten) Infektion, aber doch vor der Erkrankung, zumindest vor der neurologischen Komplikation der Erkrankung. Man darf die Hypothese aufstellen, dass das erste Krankheitsstadium der Virämie eine rasche Boosterung der Immunantwort bewirkt. 
Tabelle 1

FSME-Titer bei fälliger Auffrischimpfung.

\begin{tabular}{|c|c|c|c|c|c|}
\hline Proband & Alter & Anzahl Impfungen & Jahre seit letzter Impfung & Titerhöhe MOC & Labor \\
\hline \multicolumn{6}{|c|}{ Praxis Dr. K. } \\
\hline K.A. & 53 & 4 & 6 & 5,4 & EL Weber \\
\hline K.C. & 35 & 8 & 3 & 31,5 & EL Weber \\
\hline K.M. & 35 & 7 & 5 & 53,3 & EL Weber \\
\hline L. N. & 19 & 4 & 4 & 43,8 & EL Weber \\
\hline R.E. & 76 & 4 & 5 & 1,6 & EL Weber \\
\hline S.D. & 23 & 3 & 5 & 3,6 & EL Weber \\
\hline S.H. & 37 & 4 & 11 & 2,9 & EL Weber \\
\hline S.K. & 19 & 3 & 5 & 23,6 & EL Weber \\
\hline S.L. & 20 & 3 & 5 & 12,3 & EL Weber \\
\hline S.R. & 24 & 3 & 5 & 17,6 & EL Weber \\
\hline V.E. & 79 & 4 & 4 & 0,9 & EL Weber \\
\hline \multicolumn{6}{|c|}{ Praxis Dr. F. } \\
\hline S.E. & 48 & 3 & 4 & 7 & EL Weber \\
\hline R. T. & 30 & 3 & 5 & 5,49 & Labor Krech \\
\hline B.M. & 40 & 4 & 3 & 23 & EL Weber \\
\hline K.W. & 80 & 3 & 6 & 4,83 & Labor Krech \\
\hline S.S. & 40 & 6 & 3 & 24,8 & EL Weber \\
\hline \multicolumn{6}{|c|}{ Praxis Dr. H. } \\
\hline L.R. & 66 & 3 & 4 & 2,7 & EL Weber \\
\hline W.S. & 72 & 3 & 3 & 9,1 & EL Weber \\
\hline A.E. & 55 & 3 & 3 & 3,2 & EL Weber \\
\hline A.J. & 40 & 3 & 3 & 17,9 & EL Weber \\
\hline M.E. & 68 & 4 & 3 & 7 & EL Weber \\
\hline D. C. & 14 & 3 & 3 & 10,9 & EL Weber \\
\hline U.T. & 35 & 3 & 3 & 12,3 & EL Weber \\
\hline S.L. & 50 & 3 & 3 & 7,7 & EL Weber \\
\hline N.L. & 19 & 3 & 3 & 53,4 & EL Weber \\
\hline M.K. & 43 & 3 & 3 & 9,2 & EL Weber \\
\hline T.K. & 62 & 3 & 4 & 3,2 & EL Weber \\
\hline H.K. & 36 & 3 & 7 & 19,7 & EL Weber \\
\hline H.K. & 69 & 4 & 4 & 16,8 & EL Weber \\
\hline \multicolumn{6}{|c|}{ Praxis Dr. W. } \\
\hline W.G. & 49 & 3 & 3 & $9,0^{*}$ & Logolab \\
\hline T.P. & 47 & 4 & 4 & $4,5^{*}$ & Logolab \\
\hline S.W. & 48 & 3 & 3 & $2,6^{*}$ & Logolab \\
\hline M.G. & 42 & 3 & 2 & $12,3^{*}$ & Logolab \\
\hline S.M. & 51 & 4 & 3 & $12,6^{*}$ & Logolab \\
\hline B.M. & 55 & 3 & 6 & $8,8^{*}$ & Logolab \\
\hline \multicolumn{6}{|c|}{ Praxis Dr. G. } \\
\hline E. B. & 59 & 6 & 3 & $2^{*}$ & Logolab \\
\hline R. H. & 51 & 4 & 3 & $15^{*}$ & Logolab \\
\hline H.E. & 47 & 5 & 3 & $14,8^{*}$ & Logolab \\
\hline E.M. & 74 & 8 & 3 & $60,3^{*}$ & Logolab \\
\hline R.M. & 66 & 5 & 3 & $17,4^{*}$ & Logolab \\
\hline
\end{tabular}




\begin{tabular}{|c|c|c|c|c|c|}
\hline Proband & Alter & Anzahl Impfungen & Jahre seit letzter Impfung & Titerhöhe MOC & Labor \\
\hline R. H. & 51 & 6 & 3 & $15^{*}$ & Logolab \\
\hline L.O. & 56 & 5 & 3 & $12,6^{*}$ & Logolab \\
\hline R. O. & 24 & 3 & 3 & $19,5^{*}$ & Logolab \\
\hline E.M. & 64 & 7 & 3 & $7,9^{*}$ & Logolab \\
\hline E.H. & 59 & 5 & 3 & $7,8^{*}$ & Logolab \\
\hline W.O. & 58 & 5 & 3 & $56,1^{*}$ & Logolab \\
\hline W.H. & 78 & 6 & 3 & $1,2^{*}$ & Logolab \\
\hline
\end{tabular}

\section{Epidemiologie und Nutzen der FSME-Impfung}

Von 1989 bis 2002 wurden dem BAG 998 FSMEErkrankungsfälle gemeldet, $80 \%$ mit einer neurologischen Symptomatik. Aufgrund der Ausdehnung des FSME-Endemiegebiets in der Schweiz schätze ich 1 Million theoretisch exponierte Personen. Damit ergibt sich, dass das Risiko einer FSME-Infektion für die exponierte Bevölkerung 1:1000 beträgt während einer Beobachtungszeit von 14 Jahren. In der gleichen Zeitperiode wurden 18 Erkrankungsfälle von Geimpften gemeldet. 8 davon hatten eine vollständige Grundimmunisierung erhalten (3 oder mehr Impfungen). Seit 1989 sind in der Schweiz etwa 250000 Personen geimpft worden (rund 1 Million vertriebene Impfdosen). Das bedeutet 1 Impfversager auf 30000 lege artis geimpfte Personen. Das Risiko für einen Geimpften, an FSME zu erkranken, wird also um den Faktor 30 verkleinert. Wenn man berücksichtigt, dass die Geimpften als die eigentlichen Risikopersonen eine deutlich höhere A-priori-Wahrscheinlichkeit hatten, an FSME zu erkranken, so dürfte die tatsächliche Risikoreduktion noch um einiges höher liegen und möglicherweise den Faktor 100 übersteigen. Die FSME-Impfung darf also als hochwirksam angesehen werden.

\section{Risiken der Impfung}

«Es wurden der SANZ und dem BAG deutlich häufiger Nebenwirkungen bezüglich der FSMEImpfung gemeldet als nach anderen Impfungen: bezogen auf die in der Schweiz vertriebenen Impfdosen mit einer Häufigkeit, die 2- bis 10mal höher war im Vergleich mit anderen Impfungen.
Wenn man berücksichtigt, dass bei Kombinationsimpfungen eine Impfkomplikation jeweils allen Einzelkomponenten zugeordnet wird, so ergibt sich ein noch grösserer Häufigkeitsunterschied. Dies gilt insbesondere auch für Meldungen von neurologischen Nebenwirkungen. Die Kausalität bleibt aber offen.» [1].

2002 teilte die Schweizerische Arzneimittelnebenwirkungszentrale SANZ mit, dass zwischen 1987 und 2000 insgesamt 68 Nebenwirkungsmeldungen nach FSME eingegangen waren [2]. 33 davon betrafen neurologische Störungen. Bei 12 Meldungen, also bei jedem dritten Fall, handelte es sich um eine schwerwiegende Komplikation mit Notwendigkeit einer Hospitalisation. Da die Fälle im Spontanmeldesystem erfasst wurden, muss mit einer erheblichen Dunkelziffer nicht gemeldeter oder nicht einmal erkannter Nebenwirkungen gerechnet werden. Das BAG besitzt eigene Zahlen mit Meldungen von Impfkomplikationen. Diese sind aber noch nicht verifiziert und nicht zur Publikation bereit. Insbesondere gibt es noch keine verlässlichen Zahlen darüber, wie sich die dort gemeldeten Komplikationen aufteilen auf Erstimpfungen und Folgeimpfungen.

\section{Diskussion}

Das Enzymlabor Weber in St. Gallen hatte zu Beginn der Studie bei Titerwerten >3,0 MOC den Kommentar «immun» notiert. Somit habe ich 8 von 11 in meiner Praxis untersuchten Personen die Auffrischungsimpfung nicht empfohlen. Dr. Kälin vom Enzymlabor Weber erhielt im Zusammenhang mit meinen Recherchen von der Impfstoffherstellerfirma Baxter einen Artikel, in welchem R. Kaiser et al. [3] über den Fall 
eines 58jährigen Landwirts berichteten, der eine enzephalomyelitische Verlaufsform einer FSME mit Tetraplegie und tödlichem Ausgang erlitten hatte. Ein Monat vorher war der FSME-Titer gemessen worden zur Abklärung eines Status febrilis, der sich dann zu einem pneumonischen Infekt entwickelt hatte: 7 Jahre nach der letzten FSME-Impfung hatte man einen Titer anti-FSME von $132 \mathrm{VIEU} / \mathrm{ml}$ (entspricht 1,05 MOC) gefunden. Man hatte daraus auf eine noch wirksame Immunität gegen FSME geschlossen und auf eine Auffrischungsimpfung verzichtet. Die Autoren weisen darauf hin, dass der Elisa-Test nur bedingt zur Überprüfung des Immunstatus herangezogen werden darf wegen möglicher Kreuzreaktionen. Sie empfehlen, auf die Titermessung zur Immunitätsbestimmung zu verzichten und alle 3 Jahre eine Auffrischungsimpfung durchzuführen [3]. Aufgrund dieser Publikation verzichtet das Enzymlabor Weber St. Gallen seit dem Frühling 2003 darauf, einen Kommentar zur Immunität auf den Laborzettel zu schreiben.

Dieser einzelne Fall mit grenzwertigem Titer einen Monat vor der Erkrankung und fatalem Ausgang ist wenig aussagekräftig. Der Titer könnte falsch positiv gewesen sein, eine Kreuzreaktion bei gerade aktuellem Virusinfekt zum Zeitpunkt der Titermessung bei einem Nonresponder. Der Fall illustriert geradezu die Auffassung, dass Titerbestimmungen zur Impfkontrolle nicht während febriler oder infektiöser Zustände vorgenommen werden sollten, und legt nahe, dass der Grenzwert zur Annahme einer Immunität sicherheitshalber höher angesetzt werden sollte als der Signifikanzgrenzwert von 1,0 MOC. Das Ergebnis von 98\% signifikant positiven Titern bei unseren 47 Probanden drei und mehr Jahre nach der letzten Impfung spricht für eine ausgezeichnete immunogene Wirkung der Impfung. Zum Vergleich dient eine Prävalenzstudie für FSME, die 1992 an 969 unselektionierten und nicht gegen FSME geimpften Personen im Kanton Schaffhausen, einem bekannten Endemiegebiet für FSME, durchgeführt worden war. Die Titermessungen erfolgten anlässlich einer Blutentnahme, die aus anderen Gründen durchgeführt worden war: Nur 4,2\% der untersuchten Personen wiesen einen signifikanten Titer igG anti-FSME auf und hatten demzufolge vermutlich Kontakt mit dem FSME-Virus gehabt, ohne manifest zu erkranken [4]. Während der Impfstoffhersteller Baxter empfiehlt, alle 3 Jahre eine weitere Auffrischungsimpfung durchzuführen, verzichtet das BAG im aktuell vom Internet abrufbaren Merkblatt «Empfehlungen zur Impfung gegen Zeckenencephalitis» [5] darauf, regelmässige Auffrischungsimpfungen vorzu- schreiben und beschränkt sich auf den Hinweis, dass nach 3 Dosen bei >99\% der Fälle eine Serokonversion stattgefunden hat. Das in der Zulassung formulierte Impfschema des Herstellers gilt aber laut BAG vorläufig noch. Was die erhöhten Kosten betrifft, die entstehen bei Titermessung und nachher doch Impfung, so beobachte ich in meiner reisemedizinischen Praxis, dass in Analogie Personen, die vor 1945 geboren wurden und daher mit rund 30 Prozent Wahrscheinlichkeit eine auf natürlichem Weg erworbene Immunität gegen Hepatitis A aufweisen, fast ausnahmslos bereit sind, zuerst den Titer anti HAV bestimmen zu lassen vor einer Impfung gegen Hepatitis A.

\section{Vorschlag für eine rationale FSME-Impfung}

Grundimmunisierung wie bisher zum Zeitpunkt 0, 1 und 12 Monate. 3 Jahre später Titerkontrolle. Falls der Titer höher als 5,0 MOC ist und somit ein klar positives Resultat mit grosser Sicherheitsreserve vorliegt, Verzicht auf Impfung und Empfehlung einer nächsten Titerkontrolle in weiteren 3-5 Jahren. Aufgrund unserer 47 Fälle darf zum Zeitpunkt der fälligen Auffrischungsimpfung bei rund drei Vierteln mit einem Titerresultat über 5,0 MOC gerechnet werden. Während eine (nochmals verbesserte) zusätzliche Risikoreduktion durch immer fortdauernde Auffrischungsimpfungen trotz vorhandenem hohem Titer nicht belegt ist, muss bei jeder folgenden Auffrischungsimpfung mit einem gewissen Risiko von insbesondere neurologischen Impfkomplikationen gerechnet werden. Den gegen FSME impfenden Ärzten wird nahegelegt, in Zukunft mit besonderer Sorgfalt mögliche Impfversager mit dem zuletzt vor der Erkrankung gemessenen Titer zu melden. Damit werden wir mit den Jahren feststellen, ob eine neurologische FSME-Erkrankung trotz hohem Titer igG anti-FSME (>5,0 MOC) zustande kommen kann. Es wäre zu begrüssen, wenn in Zukunft auch sämtliche Meldungen über vermutete Impfkomplikationen beim BAG zusammenlaufen würden. Aus epidemiologischer Sicht lässt sich ein grösserer Benefit erwarten, wenn mit noch mehr Anstrengung als bisher die überhaupt nicht geimpfte Risikobevölkerung für eine Grundimmunisierung gewonnen wird. Die Empfehlung des Herstellers, alle 3 Jahre eine Auffrischungsimpfung durchzuführen, ist vermutlich übertrieben und erleichtert den Entschluss von Risikopersonen nicht, überhaupt mit einer Impfung zu beginnen. 
Dank

Ich bedanke mich bei meinen vier Kollegen Dr. Peter Flubacher, Dr. Hans-Jörg Graf, Dr. Gerold Honegger und Dr. Reto Winkler für das Mitmachen beim Erheben der Titerzahlen. Herrn Dr. Hanspeter Zimmermann vom Bundesamt für Gesundheit BAG, Abteilung Epidemiologie und Infektionskrankheiten, Sektion Impfprogramme, danke ich für die epidemiologischen Daten zu FSME in der Schweiz sowie für die äusserst hilfreiche Korrespondenz zum Thema. Herrn Dr. Christoph Hatz vom Schweiz. Tropeninstitut Basel für die konstruktiven Kommentare und kritischen Einwände, Herrn Dr. Matthias Kälin vom Enzymlabor Weber St. Gallen für die Mithilfe und Beratung im Zusammenhang mit den Titerzahlen, Herrn Dr. Beat Schmid für Ratschläge und das Manuskript über die Prävalenz der FSME im Kanton Schaffhausen sowie Herrn Dr. Ueli Grüninger, Geschäftsführer Kollegium für Hausarztmedizin, und Herrn PD Dr. Sigmund Rüttimann, Chefarzt Medizinische Abteilung Kantonsspital Schaffhausen für Lektorat und Verbesserungen sowie wertvolle Ratschläge.

\section{Literatur}

1 Schreiben von Dr. Hans-Peter Zimmermann, Abteilung Epidemiologie und Infektionskrankheiten, BAG.

2 Koller A, Hartmann K, Fleisch F, Kuhn M. Vermutete neurologische Nebenwirkungen der FSME-Impfung: Erfahrungen der Schweizerischen Arzneinebenwirkungszentrale (SANZ). Praxis 2002;91:159-62.

3 Kaiser R, et al. Impfung gegen FSME - wie lange hält der Impfschutz und was bringt die Antikörperbestimmung zur Überprüfung der Immunitätslage. Zeitschrift für Allgemeinmedizin ZFA 1999:373-4.

4 Fröhlich T, Schmid B. Prävalenz der stummen Infektion mit FSME-Virus und Borrelia Burgdorferi im Kanton Schaffhausen. Eine Studie aus dem Gesundheitsamt des Kantons Schaffhausen. 1992.

5 Bundesamt für Gesundheit, Schweizerische Kommission für Impffragen (März 2003). Empfehlungen zur Impfung gegen Zeckenencephalitis. Im Internet abrufbar unter: www.bag. admin.ch/infekt/publ/wissenschaft/d/fsme_empf. pdf 